\title{
Minimally Invasive Direct Anterior Approach Total Hip Arthroplasty: Facts and Myths
}

\author{
Rupal Patel* \\ Orthopedic surgeon, USA \\ *Corresponding author: Rupal Patel, Orthopedic surgeon, Community memorial health, VCU, USA
}

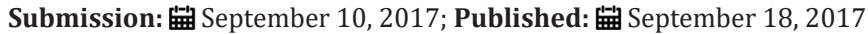

\section{Editorial}

Total hip arthroplasty is one of the most successful surgeries which improves quality of life significantly by pain relief and by improving level of activity [1]. Hip arthroplasty surgery can be performed by several different approaches. More commonly used approaches includes posterolateral (commonly known as posterior approach), anterolateral, direct lateral and direct anterior approach. Direct anterior approach (DAA) has recently gained popularity in United states for past one decade as "minimally invasive muscle sparing approach" [2]. With increasing use of internet and social media for health care information among patient population, there are some misconceptions regarding pros and cons of direct anterior approach.

According to the published survey [3] by Mayo clinic, Rochester group of arthroplasty surgeons, most of the information among their patients regarding DAA hip arthroplasty came from either friends and family or from internet. From their survey, they concluded that, The patients' perceptions are inconsistent with published data about the DAA and are likely influenced by marketing and individuals surrounding them.

Based on literature review and my own experience of performing hip arthroplasty surgery using direct anterior approach as well as posterolateral (PL) approach, below are some fact regarding direct anterior approach.

\section{Less muscle damage}

Direct anterior approach is internervous and intermuscular approach. Unlike posterolateral approach, it does not require detachment of muscles or cut any group of muscles. Though some release of capsule and some tendon release is still require to access the proximal femur in difficult exposure cases of DAA.

A comparative study suggested increase in CK level was 5.5 times more in posterolateral approach compared to DAA. The inflammatory markers were slightly decreased in DAA compared to posterolateral approach [3]. This study quantitatively confirms less muscle damage with DAA.

Study comparing postoperative MRI changes in abductor muscles, peritrochanteric bursal fluid and in tensor fascia lata suggested less fatty changes, tear or detachment in DAA approach compared to transgluteal approach [4].

\section{Functional outcome}

Several studies have suggested quicker recovery in initial postoperative period. There was no difference in functional outcome in longer term follow up.

A prospective randomized study by Barrett et al showed that DAA patients performed better in the immediate post-operative period with lower Visual Analog Scale pain scores on post-operative day one, more subjects climbing stairs and walking unlimited distances at six weeks and higher HoOS Symptoms scores at three months. However, there were no significant differences at later time points $[5,6]$.

Some authors have argued quicker recovery following direct anterior approach was patient selection bias. Most of the surgeons selected lower BMI patients for direct anterior approach during their initial cases.

\section{Dislocation rate}

For total hip arthroplasty, approaches involving anterior arthrotomy have been proven to be having less dislocation rate compared to posterior arthrotomy approach.

Some studies have claimed that the acetabular cup placement was more accurate in Lewinek safe zone with direct anterior approach compared to other approaches. Use of intra operative use of fluoroscopy during acetabular reaming and implant placement may influence better implants placement [7].

A study suggested that it is safe to remove post operative hip precautions after anterior approach hip arthroplasty [8]. Not having to observe hip precautions after total hip arthroplasty also facilitate rehabilitation during early post operative period.

\section{Shorter hospital stay}

DAA is claimed to be associated with shorter hospital stay. Initial quicker rehabilitation can be the reason for shorter hospital stay but it might have patient selection bias as well [9]. 


\section{Nerve injury}

Incidence of lateral femoral cutaneous nerve (LFCN) has been reported from $9-41 \%$ in various studies. Studies have reported the symptoms improve gradually in large number of patients but it may persist in few cases. Almost all studies have suggested that LFCN injury does not affect functional outcome of total hip arthroplasty $[9,10]$.

\section{Intra operative blood loss, fracture and operative time}

Increased intra operative blood loss, incidence of greater trochanter or calcar fracture and increased operative time has been reported with direct anterior hip arthroplasty by several studies. All these complications rate is higher during "learning curve" period while surgeon is adopting to this approach. [11] Studies have suggested incidence of these complications decrease to have no significant difference compared to other approaches for surgeons who have crossed their learning curve.

In summery, benefits of DAA total hip arthroplasty includes possible quicker recovery during initial post operative period, less dislocation rate and more accurate implant position. Downsides of this approach includes higher incidence of lateral femoral cutaneous nerve injury, incidence of femur fracture and increase blood loss during learning curve period.

Surgeons intend to adopt this approach should be mindful of stiff learning curve and higher rate of complications during initial cases. Typical learning curve involves 40 - 60 cases according to Moskal et al. [10] and Lombardi et al. [11].

Cadaveric training, surgeon to surgeon education, educative surgical technique videos and using conservative patient selection during initial period is recommended while attempt to adopt direct anterior approach total hip arthroplasty.

\section{References}

1. Padua R, Romanini E, Gillio A, Bondì R, Ceccarelli E, et al. (2005) Healthrelated quality of life after hip replacement. Journal of Orthopaedics and Traumatology 6(1): 10-14.
2. Trousdale WH, Taunton MJ, Mabry TM, Abdel MP, Trousdale RT (2017) Patient Perceptions of the Direct Anterior Hip Arthroplasty. Journal of arthroplasty 32(4): 1164-1170.

3. Bergin PF, Doppelt JD, Kephart CJ, Benke MT, Graeter JH, et al. (2011) Comparison of minimally invasive direct anterior versus posterior total hip arthroplasty based on inflammation and muscle damage markers. J Bone Joint Surg Am 93(15): 1392-1398.

4. Bremer AK, Kalberer F, Pfirrmann CW, Dora C (2011) Soft-tissue changes in hip abductor muscles and tendons after total hip replacement: comparison between the direct anterior and the transgluteal approaches. J Bone Joint Surg Br 93(7): 886-889.

5. Barrett WP, Turner SE, Leopold JP (2013) Prospective randomized study of direct anterior vs postero-lateral approach for total hip arthroplasty. J Arthroplasty 28(9): 1634-1638.

6. Beamer BS, Morgan JH, Barr C, Weaver MJ, Vrahas MS (2014) Does fluoroscopy improve acetabular component placement in total hip arthroplasty? Clin Orthop Relat Res 472(12): 3953-3962.

7. Restrepo C, Mortazavi SM, Brothers J, Parvizi J, Rothman RH (2011) Hip Dislocation: Are Hip Precautions Necessary in Anterior Approaches? Clin Orthop Relat Res 469(2): 417-422.

8. Martin CT, Pugely AJ, Gao Y, Clark CR (2013) A Comparison of Hospital Length of Stay and Short-term Morbidity Between the Anterior and the Posterior Approaches to Total Hip Arthroplasty. J Arthroplasty 28(5): 849-854.

9. Ozaki Y, Homma Y, Baba T, Sano K, Desroches A, et al.(2017) Spontaneous healing of lateral femoral cutaneous nerve injury and improved quality of life after total hip arthroplasty via a direct anterior approach. Journal of orthopedic surgery $25(1)$.

10. Moskal JT, Capps SG, Scanelli JA. (2013) Anterior muscle sparing approach for total hip arthroplasty. World J Orthop 4(1): 12-18.

11.Seng BE, Berend KR, Ajluni AF, Lombardi AV (2009) Anterior-supine minimally invasive total hip arthroplasty: defining the learning curve. Orthopedic clinic of North America 40(3): 343-350. 